\title{
INDIRECT CIRCULAR TRANSFORMATIONS AND MIXED GROUPS.
}

\author{
BY PROFESSOR H. B. NEWSON.
}

(Read before the American Mathematical Society, February 23, 1901.)

1. In a paper* entitled "Continuous groups of circular transformations," the writer gave a list of the continuous groups of circular transformations in the plane and their chief properties. That paper treated only of direct transformations. The present paper is supplementary to the former, and deals with indirect circular transformations and the mixed groups obtained by combining these with the direct transformations.

I. Properties of Indirect Circular Transformations.

2. Certain fundamental properties of indirect circular transformations were developed by Fricke and published $\dagger$ in 1890. I have seen no paper of more recent date dealing with the subject. Let $T$ be the symbol of a direct, and $\bar{T}$ the symbol of an indirect, circular transformation. Fricke's results, which form the starting point of this paper, may be stated as follows:

The second power of $\bar{T}$ is a direct circular transformation, which is either hyperbolic, parabolic or elliptic. $\bar{T}$ leaves invariant two real points, one real point, or no real points, according as its second power is hyperbolic, parabolic, or elliptic. There are three varieties of indirect transformations, viz., the hyperbolic, parabolic, and elliptic, designated by $h T, \overline{p T}$ and $\overline{e T}$, respectively, distinguished according to the character of their second powers and hence also according to the number of their invariant points. $\overline{h T}$ and $\overline{h T^{2}}$ leave invariant the same pair of invariant points $A$ and $A^{\prime} ; \overline{p T}$ and $\overline{p T^{2}}$ have the same invariant point $A$; $\overline{e T}$ has no invariant point, but interchanges the pair of points which $e T^{2}$ leaves invariant. An indirect transformation of period two is an inversion of the plane with respect to a real or pure imaginary circle. Other properties of $\bar{T}$ will now be developed.

* Bulletin (2), vol. 4, pp. 107-131 (Dec., 1897).

† Klein-Fricke's Modulfunctionen, vol. 1, pp. 196-207. 
3. Since $h T$ transforms circles into circles and leaves invariant a pair of points $A, A^{\prime}$, it must transform into itself the hyperbolic system of circles through $A$ and $A^{\prime}$; since $h T^{2}$ leaves invariant every circle of the system through $A$ and $A^{\prime}$, one effect of $\overline{h T}$ must be to interchange in pairs the circles of this hyperbolic system. Such an interchange can be effected only by an inversion of the plane with respect to $\bar{C}$, one of the circles of the system. From these conditions it follows that an indirect transformation $\overline{h T}$ must be the resultant of a direct transformation $h T$ and an inversion $I$ on one of the path curves of $h T$.

The inversion $I$ leaves invariant every point on $\bar{C}$ and every circle orthogonal to $\bar{C}$; it also produces an involutoric transformation, $k=-1$, in every circle orthogonal to $\bar{C}$. The hyperbolic system of circles through $A$ and $A^{\prime}$ contains one and only one circle $C$ orthogonal to $\bar{C}$. Since $\bar{C}$ and $C$ remain invariant under both $h T$ and $I$, they must also be invariant under their resultant $h T$. Hence, an indirect hyperbolic transformation $\overline{h T}$ leaves invariant a pair of points $\left(A A^{\prime}\right)$ and a pair of orthogonal circles intersecting in $A$ and $A^{\prime}$.

If $k$ be the cross ratio of the direct transformation $\dot{n} T$, then $h T$ produces along the circles $\bar{C}$ and $C$ one dimensional hyperbolic transformations characterized by $k$ and $-k$ respectively. Two circles through $A$ and $A^{\prime}$ making equal angles with $\bar{C}$ are interchanged by $\overline{h T}$. Thus the second power of $\overline{h T}$ leaves invariant all circles through $A$ and $A^{\prime}$ and is therefore a direct transformation of the hyperbolic kind.

There are evidently $\infty^{1}$ indirect transformations $\overline{h T}$, one for each real value of $k$, each of which has the same fundamental invariant figure. When $k=1, \overline{h T}$ reduces to the inversion with respect to $\bar{C}$; when $k=-1$, it becomes the inversion with respect to $C$.

4. By a course of reasoning similar to the above we infer the following properties of an indirect parabolic transformation : The effect of an indirect parabolic transformation $\overline{p T}$ on the points of the plane is equivalent to a direct parabolic transformation with constant $\alpha$, whereby all points in the plane are moved along their path curves, followed by an inversion with respect to $\bar{C}$, one of these path curves. Hence $\overline{p T}$ leaves invariant a single point $A$ and one circle $\bar{C}$ through $A$. Along $\bar{C}$ it produces a one dimensional para- 
bolic transformation whose constant is $\alpha$. Two circles touching $\bar{C}$ at $A$ and making equal angles with $\bar{C}$ are interchanged. There is evidently a different transformation on the same invariant figure for each real value of $\alpha$. The transformation of this system for which $\alpha=0$ is the inversion of the plane with respect to $\bar{C}$. Thus, an indirect parabolic transformation $\bar{p} \bar{T}$ leaves invariant a single point $A$ and $a$ circle through $A$ and produces along the circle a one dimensional parabolic transformation.

5. Similarly we see that an indirect elliptic transformation $\overline{e T}$ is the resultant of a direct elliptic transformation $e T$ and an inversion on one of its path curves. $e T$ leaves invariant two points $A$ and $A^{\prime}$ and moves all points in the plane along circular path curves around $A$ and $A^{\prime}$. Such a transformation followed by an inversion on $\bar{C}$, one of these path curves, results in $\overline{e T}$. $A$ and $A^{\prime}$ being inverse points with respect to every path curve of the system are interchanged by $\overline{e T}$; so that $\bar{C}$ is the only invariant figure of $\overline{e T}$. The path curves within $\bar{C}$ are interchanged with those without $\bar{C}$ in pairs. $\quad \bar{T}$ produces along $\bar{C}$ an elliptic transformation with cross ratio $e^{i \theta}$. There are $\infty^{1}$ indirect elliptic transformations leaving $\bar{C}$ invariant and interchanging $A$ and $A^{\prime}$, one for each value of $\theta$. The transformation corresponding to $\theta=0$ is the inversion on $\bar{C}$.

The circle $\bar{C}$ is a path curve of $e T$ and hence may be either real or a pure imaginary circle, $i$. e., without real points. A real inversion may take place about an imaginary circle as well as about a real circle. In the hyperbolic and parabolic cases inversion about an imaginary path curve does not occur for the reason that every path curve has on it at least one real point. Thus we see that an indirect elliptic transformation eT leaves invariant a single circle and produces along that circle a one dimensional elliptic transformation.

Our discussion may be summed up in the following theorem :

Theorem 1. Every indirect circular transformation of the plane is the resuitant of a direct non-loxodromic transformation and an inversion on one of its path curves.

\section{Mixed Groups of Circular Trangformations.}

6. The Mixed Group $m G_{6}$. The indirect circular transformations whose properties have just been developed do 
not form continuous groups, but taken with direct transformation form mixed groups. There are $\infty^{6}$ direct and $\infty^{6}$ indirect circular transformations of the plane and the aggregate of all these forms the mixed group $m G_{6}$. This general group contains many subgroups which are also mixed groups; these we now proceed to examine.

7. The Mixed Group $m G_{2}\left(A A^{\prime}\right)$. We seek the aggregate of all transformations both direct and indirect which leave two points $A$ and $A^{\prime}$ separately invariant, and also those which interchange them. Two points $A$ and $A^{\prime}$ remain invariant under $\infty^{2}$ direct transformations which form the continuous group $G_{2}\left(A A^{\prime}\right)$. Let $P$ and $Q$ be two points harmonic to $A$ and $A^{\prime}$ (whence $P Q A A^{\prime}$ are four points on a circle). The involutoric transformation in the group $G_{2}(P Q)$ interchanges $A$ and $A^{\prime}$. Since $\infty^{2}$ pairs of points may be chosen harmonic to $A$ and $A^{\prime}$, we see that there are $\infty^{2}$ direct involutoric transformations interchanging $A$ and $A^{\prime}$.

The indirect transformations which leave $A$ and $A^{\prime}$ separately invariant are all of the hyperbolic variety. There are $\infty^{1}$ circles through $A$ and $A^{\prime}$; each of these with the invariant points $A$ and $A^{\prime}$ is the invariant figure of $\infty^{1}$ indirect hyperbolic transformations. Thus we see that there are $\infty^{2}$ indirect hyperbolic transformations leaving $A$ and $A^{\prime}$ separately invariant.

The indirect transformations which interchange $A$ and $A^{\prime}$ are all of the elliptic variety. There are $\infty^{1}$ circles in the elliptic system of which $A$ and $A^{\prime}$ are the vanishing points. Each of these circles is invariant under $\infty^{1}$ indirect elliptic transformations which interchange $A$ and $A^{\prime}$. 'Thus we see there are $\infty^{2}$ indirect elliptic transformations interchanging $A$ and $A^{\prime}$.

The aggregate of all direct and indirect transformations which leave a point pair invariant forms a mixed group $m G_{2}\left(A A^{\prime}\right)$. From the above discussion we see that it contains two kinds of direct and two kinds of indirect transformations. The decomposition of $m G_{2}\left(A A^{\prime}\right)$ into subgroups will be discussed in articles 10 and 11 .

Theorem 2. The mixed group $m G_{2}\left(A A^{\prime}\right)$ contains $\infty^{2} d i$ rect transformations (which form the continuous group $G_{2}\left(A A^{\prime}\right)$ ), $\infty^{2}$ direct involutoric transformations, $\infty^{2}$ indirect hyperbolic, and $\infty^{2}$ indirect elliptic transformations.

8. The Mixed Grou p $m p G_{3}(A)$. We seek next the aggre. gate of all parabolic transformations both direct and indirect which leave a single point $A$ invariant. We know that $A$ remains invariant under $\infty^{2}$ direct parabolic transformations which form the continuous group $G_{2}(A)$. The 
indirect transformations which leave a single point $A$ invariant are all of the parabolic type. There are $\infty^{2}$ circles through $A$; each of these circles with the invariant point $A$ is the invariant figure of $\infty^{1}$ indirect parabolic transformations. Hence there are $\infty^{3}$ indirect parabolic transformations leaving the point $A$ invariant. The aggregate of all parabolic transformations both direct and indirect which leave $A$ invariant forms a mixed group, $m p G_{3}(A)$. Its subgroups will be investigated in article 12 .

Theorem 3. The mixed group $m p G_{3}(A)$ contains $\infty^{3}$ indirect and only $\infty^{2}$ direct parabolic transformations.

9. The Mixed Group $m G_{4}(A)$. We know that there are $\infty$ direct transformations leaving a single point invariant and these form a continuous group $G_{4}(A)$. There are also $\infty$ indirect hyperbolic transformations having one invariant point at $A$; the second invariant point $A^{\prime}$ is in turn every point in the plane. There are also $\infty^{3}$ indirect parabolic transformations leaving $A$ invariant. The aggregate of all these transformations both direct and indirect forms a mixed group $m G_{4}(A)$. This mixed group contains no indirect elliptic transformations.

Theorem 4. The mixed group $m G_{4}(A)$ contains $\infty^{4}$ direct, $\infty^{4}$ indirect hyperbolic, and $\infty^{3}$ indirect parabolic transformations.

10. The Mixed Group $m h G_{1}\left(A A^{\prime} \bar{C}\right)$. The continuous group $G_{2}\left(A A^{\prime}\right)$ contains one continuous subgroup of hyperbolic transformations $h G_{1}\left(A A^{\prime}\right)$, whose invariant path curves are the circles of the hyperbolic system determined by $A$ and $A^{\prime}$. Each of these circles passing through $A$ and $A^{\prime}$ is invariant under $\infty^{1}$ indirect hyperbolic transformations. Let $\bar{C}$ be one of these circles; then $\bar{C}$ is invariant under $\infty^{1}$ direct and also $\infty^{1}$ indirect hyperbolic transformations. These two systems form the mixed group $m h G_{1}\left(A A^{\prime} \bar{C}\right)$.

Within the mixed group $m G_{2}\left(A A^{\prime}\right)$ there are $\infty^{1}$ such subgroups, one for each circle of the hyperbolic system determined by $A$ and $A^{\prime}$. All of these mixed groups contain the same continuous group of direct hyperbolic transformations $h G_{1}\left(A A^{\prime}\right)$.

Theorem 5. The mixed group $m G_{2}\left(A A^{\prime}\right)$ contains $\infty^{1}$ mixed subgroups $m h G_{1}\left(A A^{\prime} \bar{C}\right)$, all of which contain the same continuous group $h G_{1}\left(A A^{\prime}\right)$.

11. The Mixed Group $m e G_{1}\left(A A^{\prime} \bar{C}\right)$. The continuous group $G_{2}\left(A A^{\prime}\right)$ contains one continuous subgroup of elliptic transformations $e G_{1}\left(A A^{\prime}\right)$ whose invariant path curves are the $\infty^{1}$ circles having $A$ and $A^{\prime}$ for a pair of inverse points. 
Each circle of this elliptic system is invariant under $\infty^{1}$ indirect elliptic transformations. Let $\bar{C}$ be one of these circles, then $\bar{C}$ is invariant under $\infty^{1}$ direct and also $\infty^{1}$ indirect elliptic transformations. These two systems form the mixed group $m e G_{1}\left(A A^{\prime} \bar{C}\right)$.

Within the mixed group $m G_{2}\left(A A^{\prime}\right)$ there are $\infty^{1}$ such subgroups, one for each circle of the elliptic system determined by $A$ and $A^{\prime}$. Each of these mixed groups contains the samecontinuous group of elliptic transformations, $e G_{1}\left(A A^{\prime}\right)$.

Theorem 6. The mixed group $m G_{2}\left(A A^{\prime}\right)$ contains $\infty^{1}$ mixed subgroups me $G_{1}\left(A A^{\prime} \bar{C}\right)$, all of which contain the same continuous group e $G_{1}\left(A A^{\prime}\right)$.

12. The Mixed Groups $m p G_{2}(A)$ and $m p G_{1}(A \bar{C})$. The continuous group $p G_{2}(A)$ contains $\infty^{1}$ continuous groups $p G_{1}(A)$. The path curves of one of these subgroups form a parabolic system of circles through $A$. Each circle $\bar{C}$ of this parabolic system is invariant under $\infty^{1}$ indirect parabolic transformations; consequently the circle $\bar{C}$ and the point $A$ on it are invariant under $\infty^{1}$ direct and also $\infty^{1}$ indirect parabolic transformations, which therefore form a mixed group $m p G_{1}(A \bar{C})$. The group $m p G_{3}(A)$ contains $\infty^{2}$ such groups $m p G_{1}(A \bar{C})$, one for each circle through $A$.

The aggregate of all the groups $m p G_{1}(A \bar{C})$ whose invariant circles form a parabolic system of circles through $A$ constitute a group $m p G_{2}(A)$. For this parabolic system of circles remains invariant under all of these $\infty^{2}$ transformations ; the system as a whole remains invariant though the individual circles of the system are not necessarily invariant under all the transformations of the group. The $\infty^{1}$ subgroups $m p G_{1}(A \bar{C})$ of $m p G_{2}(A)$ all contain the same continuous subgroups $p G_{1}(A)$.

Theorem 7. The mixed group $m p G_{3}(A)$ contains $\infty^{1}$ mixed subgroups $m p G_{2}(A)$ and each of these contains $\mid \infty^{1}$ mixed subgroups $m p G_{1}(A \bar{C})$. All subgroups $m p G_{1}(A \bar{C})$ of $m p G_{2}(\boldsymbol{A})$ contain the same continuous subgroup $p G_{1}(A)$.

13. The Mixed Group $m G_{3}(\bar{C})$. Let $\bar{C}$ be any real circle in the plane; we know that $\bar{C}$ is invariant under $\infty^{3}$ direct transformations which form a continuous group $G_{3}(\bar{C})$. Every direct transformation $T$ in this continuous group followed by an inversion on the circle $\bar{C}$ results in an indirect transformation $\bar{T}$, and the aggregate of all these transformations both direct and indirect forms a mixed group $m G_{3}(\bar{C})$. 
Every pair of points on $\bar{C}$ are the invariant points of a mixed group of hyperbolic transformations $m h G_{1}\left(A A^{\prime} \bar{C}\right)$, leaving $\bar{C}$ invariant; hence $m G_{3}(\bar{C})$ contains $\infty^{2}$ such mixed groups of hyperbolic transformations. Every point on $\bar{C}$ is the invariant point of a mixed group of parabolic transformations $m p G_{1}(A \bar{C})$, leaving $\bar{C}$ invariant; hence $m G_{3}(\bar{C})$ contains $\infty^{1}$ such mixed subgroups of parabolic transformations. Every pair of points inverse with respect to $\bar{C}$ are interchanged or remain invariant under the transformations of a mixed elliptic group $m e G_{1}\left(A A^{\prime} C^{\prime}\right)$; hence $m G_{3}(\bar{C})$ contains $\infty^{2}$ such mixed subgroups of elliptic transformations.

Theorem 8. The mixed group $m G_{3}(\bar{C})$ contains $\infty^{2}$ mixed subgroups $m h G_{1}\left(A A^{\prime} \bar{C}\right)$, $\infty^{2}$ mixed subgroups me $G_{1}\left(A A^{\prime} \bar{C}\right)$, and $\infty^{1}$ mixed subgroups $m p G_{1}\left(A C^{\top}\right)$.

14. The Mixed Group $m h G_{2}(\overline{A C})$. The mixed group $m G_{3}(\bar{C})$ contains $\infty^{2}$ hyperbolic subgroups $m h G_{1}\left(A A^{\prime} \bar{C}\right)$. Let the point $A$ remain fixed and let the point $A^{\prime}$ be in turn every point on $\bar{C}$. The aggregate of all the subgroups $m h G_{1}\left(A A^{\prime} \bar{C}\right)$ whose invariant points satisfy this condition forms a mixed group $m h G_{2}(A \bar{C})$. This group contains one parabolic subgroup $m p G_{1}(A \overline{C)}$, but no elliptic subgroups. The group $m G_{3}(\bar{C})$ contains $\infty^{1}$ such subgroups $m p G_{2}(A \bar{C})$, one for each point on the circle $\bar{C}$.

Theorem 9. The group $m G_{3}(\bar{C})$ containing $\infty^{1}$ subgroups $m h G_{*}(A \bar{C})$, each of which contains hyperbolic and parabolic transformations but no elliptic transformations.

15. The Mrxed Group $m G_{3}(\overline{i \bar{C}})$. Leti $\bar{C}$ be any imaginary circle in the plane. We know that $\overline{i C}$ is invariant under $\infty^{3}$ direct elliptic transformations which form the continuous group $G_{3}(\bar{i} C)$. Every transformation in this group followed by an inversion on the circle $i \bar{C}$ results in an indirect elliptic transformation $\overline{e T}$ and the aggregate of all these transformations both direct and indirect forms the mixed group $m G_{3}(i \bar{C})$. This group evidently contains $\infty^{2}$ mixed subgroups $m e G_{1}\left(A A^{\prime} \bar{C}\right)$, one for each pair of points in the plane inverse with respect to the circle $i \bar{C}$.

Theorem 10. The group $m G_{3}(i \bar{C})$ contains only elliptic transformations; these fall into $\infty^{2}$ subgroups me $G_{1}\left(A A^{\prime} \bar{C}\right)$.

Résumé. - We have thus found a list of eleven mixed g roups 
of circular transformations of the plane. These with their appropriate symbols and invariant figures are as follows:

1. $m G_{6}$. No invariant figure.

2. $m G_{4}^{6}(A)$. A single invariant point.

3. $m p G_{3}(A)$. A single invariant point.

4. $m G_{3}(\bar{C})$. A real invariant circle.

5. $m G_{3}(i \bar{C})$. An imaginary invariant circle.

6. $m p G_{2}(A)$. A single invariant point.

7. $m G_{2}\left(A A^{\prime}\right)$. An invariant point pair.

8. $m h G_{2}(A \bar{C})$. An invariant point and a circle through it.

9. $m h G_{1}\left(A A^{\prime} \bar{C}\right)$. A pair of invariant points and a pair of orthogonal circles.

10. $m p G_{1}(A \bar{C})$. An invariant point and an invariant circle.

11. $m e G_{1}\left(A A^{\prime} C\right)$. An invariant point pair and an invariant circle.

LAWRENCE KANSAs, January 18, 1901 .

\section{PURE MATHEMATICS FOR ENGINEERING STUDENTS.}

BY PROFESSOR A. S. HATHAWAY.

(Read before the Chicago Section of the American Mathematical Society, December 28, 1900.)

\section{Its Utility.}

I HAVE had opportunity to become acquainted with the written opinions of graduates of from one to many years' standing, with regard to the benefit and utility of their instruction in all departments of technical work, and have also taken the opportunity of conferring personally with graduates in respect to my own department. In discussing this subject, I shall therefore appear, not as a special pleader for pure mathematics, but as one who proposes to present its claims in their true proportions to the other necessary work of the student.

In the first place, mathematical analysis is not so directly useful to the average engineer as the mathematician might expect. The mathematics that an engineer is obliged to use regularly is of that cut and dried form which is found tabulated in engineering handbooks of easy access, so that only a little arithmetic or algebra, and occasionally some 